\title{
The Impacts of Awareness Tools on Mutual Modelling in a Collaborative Video-Game
}

\author{
No Author Given \\ No Institute Given
}

\begin{abstract}
This paper describes the findings of an experimental research concentrating on collaboration in a multi-player video game. The overall goal is to study the cognitive impacts of the awareness tools. The focus is in finding an effect on performance as well as on the representation an individual build of what his partner knows, plans and intends to do (i.e. Mutual Modelling). Using an awareness tools has a significant effect by improving task performance. However, the players who were provided with this tool did not show any improvement of their mutual modelling. Further analysis on contrasted groups revealed that there was an effect of the awareness tool on mutual modelling for players who spent a large amount of time using the tool... .
\end{abstract}

\section{Introduction}

The challenge of today's collaborative systems is to overcome the computer limits so as to make participants and their activities visible to one another. This is called awareness: the understanding of the teammates' activities and interactions in the workspace. Awareness has recently become a new research field particularly for CSCW (Computer Supported Collaborative Work) and CSCL (Computer Supported Collaborative Learning). Being (and also remaining) aware of others is as important in everyday life as in groupware systems. The lack of information about the others in multi-user environment is addressed by providing users with tools that try to "recreate the information landscape of a real-world landscape" [1] : the awareness tools (from now on called AT in this paper). Consequently, the AT are in general supposed to enable users to offset the lack of social interactions. They also provide a more efficient team collaboration by showing information about presence (is anyone in the workspace?), identity (who is that?), location (where is an individual?), action (what is somebody doing?), etc.

In the field of Human-Computer Interaction, there have been relatively few occurrences of research concerning the empirical evaluation of awareness tools. Few studies provide data about the use of AT [2] and the usability issues of AT [3] [4]. The objective of our is to examine the impact of AT on what is called mutual modelling. Mutual modelling is the representation and the expectations that an individual constructs and maintains of what his/her partner knows, does, believes and intends to do. We have investigated whether providing peers with AT can help building more accurate mutual models and being more effective. 
It is important to investigate the cognitive benefits of mutual modelling as it can be postulated that maintaining a representation of the other's viewpoint explains some of the results of collaborative learning. As a matter of fact, collaboration effects seem produced by different kind of collaborative interactions (explanation, argumentation,...) that require to develop a shared understanding of the task. In order to detect misunderstandings and disagreement during the interactions, the subjects must maintain some representation of what their partner understands. It can be postulated that the effort for maintaining a model of the partner contributes itself to the learning process as it forces the learner to reason more deeply on the domain and to perceive the task from a second viewpoint. Now, this raises an old educational issue: if we support mutual modeling with awareness tools, do we facilitate learning and hence expect higher learning gains or, conversely, do reduce the cognitive effort and hence decrease the learning gains to be expected ? This research, by augmenting mutual modeling with awareness tools, is a first step to grasp the cognitive effects of awareness tools and mutual modeling.

\section{Methodology}

In order to reach that goal, a computer game was employed to conduct experiments. In this game, two players located in different rooms are involved in a space mission where they were required to collaborate.

\subsection{Spaceminers : a collaborative video-game}

SpaceMiners is an experimental platform in the form of a video game for running psychological experiments. It has been developed at the Geneva Interaction Lab (University of Geneva) by Yvan Bourquin, Jeremy Goslin and Thomas Wehrle.

As can be seen on figure 1 the space is represented by a grid with planets, asteroids, the spacestation (the circles on the left) and the spaceship explorer. The line shows the trajectory of a launched drone. Here, the direction is modified by the planet's gravity. The purpose of the mission represented here is to collect the largest amount of minerals located in asteroids and to bring them to the space station on the left. The score represents the number of collected minerals docked to the space stations launched by the two players. The score is influenced by several factors such as : the drone trajectory, the launch speed, the tools positions (that influence the drone trajectory), the number of asteroids in the environment, the planet positions (that modify the gravity).

Users can play in two modes that corresponds to two viewpoints : the explorer mode and the camera mode. They can switch from one mode to another by pressing a key on the joystick.

In the explorer mode, the position of the spaceship is fixed and players launch drones that pass through as many asteroids as possible on their way to the space station. Once the drones are launched players have no control over them. Their trajectory depends on the direction of the explorer and the launch speed of 
the drone controlled by the player and on the gravity of planets. Additionaly, different tools dragged into space by players could modify the drone's trajectory.

In the camera mode, players can move their camera around in space by moving the joystick. The camera is very useful to see space from another viewpoint and to place tools in space. To distinguish this mode from the other, there is no crosshair and no green border around the screen. Fig. 1 shows the space and the ship seen by the camera. Fig. 2 shows the camera as it can be seen in the explorer mode.

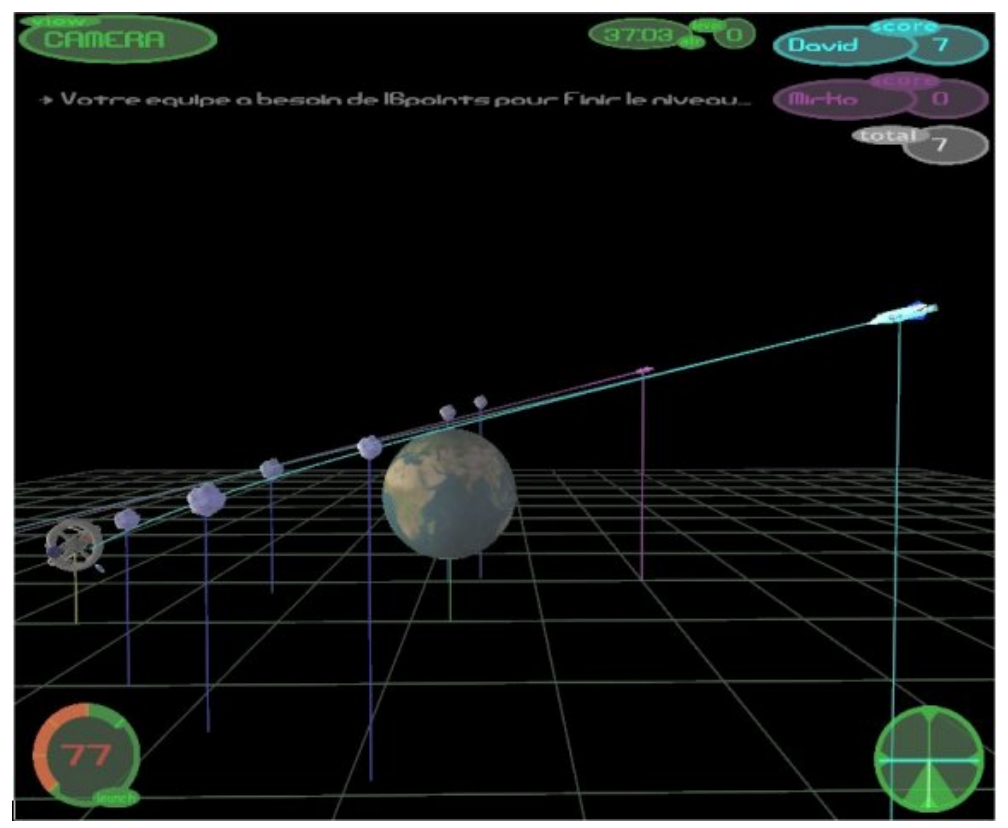

Fig. 1. the game environment made up of a planet, asteroids, two spaceships and a space station. This kind of view can be seen in the camera mode. This screenshot hence depicts the camera view (since we see the spaceship) as indicated in the upper left-hand corner. David (the player who controls the ship) manages to collect asteroids and to dock his drone to the space station. Thus he wins 7 points

In our experiment, we focused on one kind of awareness tool : the view of the partner's camera and his laser pointer as presented in Fig. 2. By seeing the camera of his partner the player can obtain awareness information about his team-mate location and gaze. Thus he could help him to drop tools into space or to adjust the trajectory of the drone the partner intends to launch. The presence or absence of this awareness tool constitutes the experimental condition of the study.

There are two tools that can be dropped in space or dragged behind the player's camera : the blackhole and the gate. The blackhole has a very high 


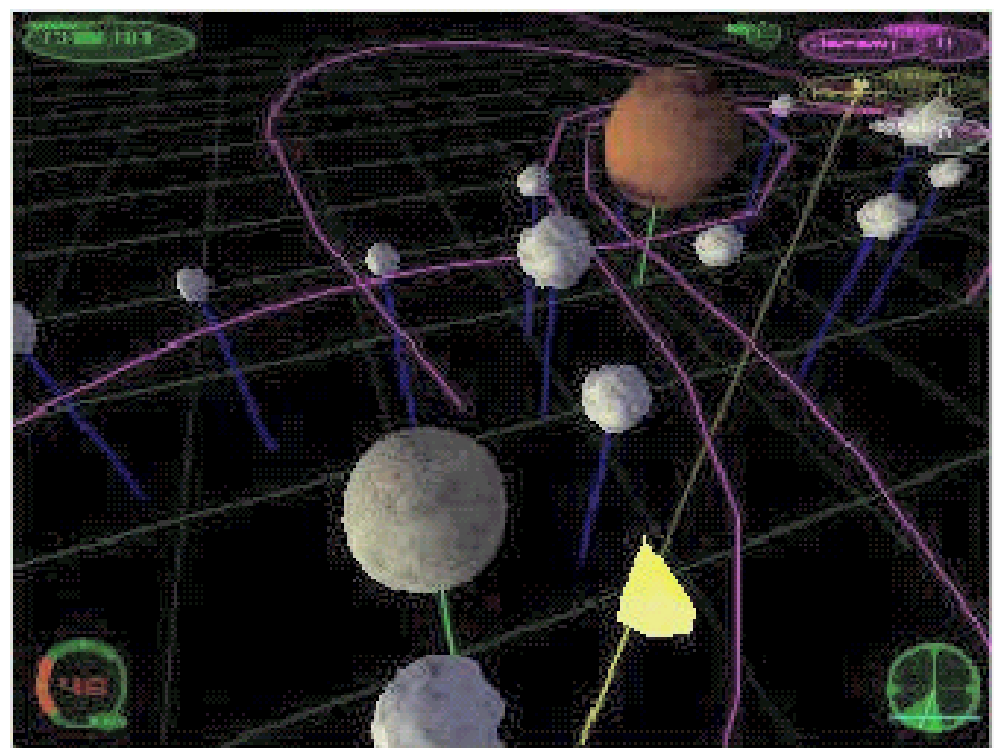

Fig. 2. view of the camera. This view can only be seen in the explorer mode (from the spaceship)

gravitational pull : it pull drones towards it. Gates are stabilised entrances to wormholes in spacetime. If two are placed in space then a gate will transfer a drone from one position in space to another instantaneously. Players sees the tools available represented in a toolbox. When someone drops a tool in space, the tool's icon is removed from the toolbox and the object stands in space just behind the player's explorer. If he wants to see it, he has to make a rotation.

The tools available depends on the level of the game. In level one, players are given no tools. In level two and three, each player has different tools in order to foster collaboration between them. In level 2, each player has only one gate. In level 3, one player has blackhole and a gate and the other has only one gate. The procedure of collaborative problem solving consists in the fact that the two players need to negotiate about the position of those tools in space. It is not possible to finish the three levels without talking to each other or deciding where to put a tool. Another collaborative behavior is to help the partner to navigate, to drop a tool, to adjust his/her shoot as well as role distribution.

SpaceMiners is a video game designed to elicit collaborative behaviour. The use of a game metaphor has the advantage that it allows the presentation of complex problem solving tasks in an enjoyable environment, thus maintaining a high level of motivation amongst subjects. However, these statements only hold for subjects that find such games enjoyable, those with little interest in games can fail to engage with the game, finding both the task and the interface difficult and confusing. 


\subsection{Variables}

The presence or absence of the awareness tool constitutes the experimental condition of the study : it is our independent variable. We used two dependent variables in order to test our hypotheses : task performance and mutual modelling. Concerning task performance, we used the pairs' score : player A's score added to player B's score. Concerning Mutual Modelling (MM), two different questionnaires allowed us to calculate an evaluation the value of MM for a pair. First, during the game and for each of the three levels, players had to answer to two multiple choice questionnaires. Those questionnaires asked them about what they are intending to do at the moment (guiding his partner, trying to understand his strategy, trying to establish a common strategy, adjusting a shoot, etc.). Then, the questionnaires asked each player about what he thinks his partner is currently doing (same propositions as the previous questionnaire). We compared the first answer of a player (about what $\mathrm{A}$ is intending to do) to the answer of his partner to the second question (about what B believes A is doing). Consequently, our evaluation of the MM accuracy is the number of common answers to those two questions. We compared whether A's prediction of B's answer matches with B's actual answer. Thanks to those MM evaluation, we could define different variables presented in Table 1.

Table 1. description of the dependent variables concerning Mutual Modelling

\begin{tabular}{ll}
\hline Evaluation & Description \\
\hline MM1 & $\begin{array}{l}\text { Mutual Modelling evaluation for the pair } \\
\text { measured in the first level } \\
\text { Mutual Modelling evaluation for the pair } \\
\text { measured in the second level } \\
\text { MM2 }\end{array}$ \\
Mutual Modelling evaluation for the pair \\
measured in the third level \\
MMg $=$ MM1+MM2+MM3 \\
Global Mutual Modelling evaluation \\
for the pair
\end{tabular}

In game level 1 , questionnaires are displayed 5 minutes after the beginning. In levels 2 and 3, questionnaires are displayed after the first time a player dropped a tool in the environment.

\subsection{Hypotheses}

We postulate three hypotheses :

Hypothesis H1 : Pairs with the awareness tool are more effective than pairs without it. As a matter of fact, the information brought by the AT can enable players to complete the task more efficiently. In order to evaluate the performance, we use the team score, sum of the two players' scores. Then we expect that the team score is higher when players have an awareness tool. 
Hypothesis H2 : Pairs with the awareness tools build more accurate model than pairs without it. The global mutual modelling evaluation (MMg) should be higher when the players have an awareness tool. The MMg is the sum of the objective evaluations of the mutual modelling of a team during the whole game, measured by the in-game questionnaires.

Hypothesis H3 : the mutual modelling accuracy improves with time: when partners learn to know each other, the representation of each others' strategies is more accurate. As a consequence, we expect MM3 to be higher than MM1.

\subsection{Participants, settings and procedure}

Participants were recruited in Geneva. We chose only men familiar in order to avoid gender bias. Experimental subjects consisted of 18 pairs $(\mathrm{N}=18)$. Those pairs were assigned to one of the experimental conditions forming two groups of 9 pairs. Participants were assigned a partner they were not familiar with. The game was played on two computers over the local network. Each player sit in front of a distinct computer located in different rooms and could communicate by voice thanks to a headset. Experiments lasted approximately 2 hours and were conducted in French. After a tutorial, players had to complete three levels. Mutual modelling questionnaires were displayed during missions 1, 2 and 3.

\section{Results}

According to hypothesis H1, the awareness tool enables pairs to increase their performance. We want to test the effectiveness of using an AT. Table 2 shows the descriptive statistics.

Table 2. descriptive statistics for hypothesis 1

\begin{tabular}{lllllll}
\hline & & N & Mean & Std Dev & Min & Max \\
\hline \multirow{2}{*}{ Score } & With AT & 9 & 258.67 & 90.80 & 127 & 360 \\
& Without AT & 9 & 175.67 & 67.48 & 51 & 235 \\
& Total & 18 & & & 51 & 360 \\
\hline
\end{tabular}

The descriptive statistics shows that pairs with AT reached higher score than the others. Besides, no pairs without AT reached the mean score of the pairs with AT. the ANOVA test confirms that there is a significant differences between the two conditions $(\mathrm{F}=4.84, \mathrm{p}=0.043)$. Therefore, our first hypothesis is validated : there is a significant effect of the awareness tool on performance. Our second hypothesis $\mathrm{H} 2$ is that the use of an awareness tool improves the mutual modelling accuracy of a pair. We assume that players with awareness tool have more accurate mutual modelling, that is to say : MMg (With AT) i MMg (Without AT). Table 3 shows the descriptive statistics. 
Table 3. descriptive statistics for hypothesis 2

\begin{tabular}{lllllll}
\hline & & N & Mean & Std Dev & Min & Max \\
\hline MMg & With AT & 9 & 1.63 & 0.48 & 1 & 2.67 \\
& Without AT & 9 & 1.58 & 0.87 & 0.83 & 3.17 \\
& Total & 18 & & & 0.83 & 3.17 \\
\hline
\end{tabular}

Table 3 shows that the MMg means are very close. It goes against our hypothesis and the ANOVA test shows that $\mathrm{H} 2$ is invalidated $(\mathrm{F}=0.02, \mathrm{p}=.889)$. The use of the AT does not improve the accuracy of the mutual modelling. The representation of one's partner strategy is not facilitated by the information conveyed by the awareness tool. Our third hypothesis assumes that there is an effect of time and collaboration on mutual modelling. At the beginning of the game, the players are not familiar with each other. We hence postulated that playing together during two hours enables them to improve the accuracy of their mutual modelling. We assume that MM3 which is the evaluation of the accuracy of the mutual modelling measured in level 3 is higher than MM1 which is the evaluations of the accuracy of the mutual modelling measured in level 1 . Table 4 shows the descriptive statistics.

Table 4. descriptive statistics for hypothesis 3

\begin{tabular}{lllllll}
\hline & & N & Mean & Std Dev & Min & Max \\
\hline MM & MM1 & 18 & 1.33 & 0.84 & 0 & 3 \\
& MM3 & 18 & 1.94 & 1.14 & 0.5 & 4 \\
Total & 36 & & & 0 & 4 \\
\hline
\end{tabular}

The descriptive statistics shows a slight difference between the means of MM1 and MM3. According to the test presented in table 4, concerning the effect of time on the accuracy of mutual modelling, it is only a trend $(\mathrm{F}=3.189, \mathrm{p}=0.084)$. We reject $\mathrm{H} 3$ with $\mathrm{p}=0.084$. Additionally, there is no effect of the presence of the awareness tool $(\mathrm{F}=0.105, \mathrm{p}=0.748)$. This result is consistent with the invalidation of our second hypothesis. There is also no interaction between time and the presence of the awareness tool $(\mathrm{F}=0.105, \mathrm{p}=0.748)$. As a consequence, this supports the argument that the increase of the accuracy of the mutual modelling is not due to the presence of the awareness tool.

\section{Discussion and further analysis}

The fact that teams in the awareness tool condition reach higher score than the others is consistent with the findings of [3] and [5]. They also found that 
awareness can be beneficial to team performance. In fact, this tool provide a continuous feedback to the partner who can see where is his team-mate. This could be extremely useful in tasks like object positioning. In such tasks, player A guided player B's movement by giving him instructions about where dropping the object. Additionally, the AT provided visual evidences about the player's location. The team is thus more effective because player A had not to verbally describe where he is and player B had not to interpret this description. As suggested by [3], the use of the awareness tool leads to transform a task from a verbal to a visual activity.

Our second hypothesis was invalidated, the representation of one's partner strategy is not facilitated by the information conveyed by the awareness tool. Presumably, those results lead us to three possible conclusions. First, the awareness tool, by showing information about the partner's locations and gaze does not improve the accuracy of the mutual modelling. Second, it is possible that the game does not require participants to maintain accurate mutual models, perhaps they do not have to build a very accurate representation of the partner's intentions. And finally, perhaps, our evaluation of mutual modelling is not very precise.

We also focused on behavioral data which were stored in the logfiles. We looked specifically at the percentage of time spent in each view (camera or spaceship) by the pairs, we could notice an interesting difference. A two-way analysis of variance conducted on contrasted groups showed that pairs in the awareness condition who spent more time in the camera mode reached higher levels of mutual modelling $(\mathrm{F}=8.02, \mathrm{p}=0.015)$ than the others. It implies that there is an effect of the awareness tool on mutual modelling only for the teams who spent a long time in the camera mode. Awareness information could help players in order to estimate their partner's strategies if the participants understand that they have to make an effort : spending an accurate time in the camera view. The team who did not spend enough time in the camera view had no benefit of the awareness tool. Our finding raises a new question : do players really used the awareness tool ? Indeed, if there is an effect of the tool on mutual modelling only for the players who used it frequently, it may be possible that only a few players in the tool condition noticed the advantage of using it.

Hypothesis 3 postulated an effect of time on the accuracy of mutual modelling. There seems to be an effect but it is not statistically significant. Our hypothesis is invalidated but the findings call for certain restrictions because of the questionnaire. Using a simple questionnaire to measure the accuracy in predicting partners answers is way too subjective. We should use a more objective method to evaluate this variable. A solution would be to analyze the redundancy (i.e. the number of times player A performs an action that player B's previously performed). 


\section{Conclusion}

We explored the field of research concerning the cognitive impacts of the awareness tools used in collaborative multi-user environments. The studies we have presented in this document address two issues : the effect of the awareness tool on performance and the impact on the mutual modelling,. So far, only some findings confirmed our hypotheses and intuitions. Some results, though seem to be contrary to expectations.

Nonetheless, those results call for certain restrictions. On the one hand, the number of participants is quite low : eighteen pairs (nine in each conditions). On the other hand, we can also have reservations about the experimental design. Furthermore, the method used to measure the accuracy of the mutual modelling may be unsuitable.

We could criticize the tool we used as well. Actually, we considered here the awareness as a simple tool that convey specific information about the participants' behavior. In fact, awareness is not only this kind of "widget", the situation is more intricate. We should reconsider the definition of awareness as a diffuse flow of information [6] : lots of different cues, signs, evidences which are combined. This flow makes sense and it is very difficult to create a tool to enable this combination.

There are several impacts for practitioners as well, for instance for CSCL. The findings provide evidence that location and gaze awareness can be useful in certain situations. Our findings could help produce recommendations for designers. As we mentioned above, the use of the awareness tool in Spaceminers leads to transform a task from a verbal to a visual activity and hence induce a quicker completion of the task. This a clear lesson for designers : instead of letting participants describing their locations or the artifacts they are talking about, an awareness tool could usefully show those kind of indications. Another interesting lesson is that designers should keep in mind that using an AT is not systematic. As we have seen in our experiment, several players did not really notice the potential of this tool. Thus, designers should provide users with usable AT and teach them their real value.

\section{References}

1. Gutwin, C. Greenberg, S. A framework of awareness for small groups in sharedworkspace groupware. (Technical Report 99-1), Department of Computer Science, University of Saskatchewan, Canada (1999)

2. Jang, C.Y., Steinfield, C., Pfaff, B. Virtual team awareness and groupware support : an evaluation of the TeamSCOPE system. International Journal of HumanComputer Studies, 56, 109-126 (2002)

3. Gutwin, C., Roseman, M., Greenberg, S. A usability study of awareness widgets in a shared workspace groupware system. In Proceedings of the ACM Conference on Computer Supported Cooperative Work, 258-267 Boston: ACM Press (1996).

4. Gutwin, C., Greenberg, S. Effects of Awareness Support on Groupware Usability In Proceedings of the Conference oh Human Factors in Computing Systems (CHI 98), 511-518. Los Angeles : ACM Press (1998) 
5. Espinosa, A., Cadiz, J., Rico-Guttierez, L., Kraut, R., Scherlis, W. Lautanbacher, G. Why Awareness Tools Must be Matched with Appropriate Tasks. In Proceedings of the Conference on Human Factors in COmputing Systems (CHI2000), 392-399. The Hague, Amsterdam ACM Press (2000)

6. Mastrogiacomo, S. Utilisation des zones de travail partages asynchrones pour amliorer la comprhension mutuelle dans les groupes de projet distribus. Thse de doctorat en informatique de gestion non publie, Ecole des Hautes Etudes Commerciales, Universit de Lausanne, Lausanne (2002). 